\title{
Research Paper: The Development and Validation of a Scale for the Activities of Daily Living in Iranian Children
}

\author{
Hossein Soltaninejad ${ }^{1}$ (D), Mehdi Alizadeh Zarei $^{2 *}$ (D), Malahat Akbarfahimi² (i), Akram Azad ${ }^{2}$ (D), Farzad Rabiei ${ }^{1}$ (i)
}

1. Department of Occupational Therapy, School of Rehabilitation, Iran University of Medical Sciences, Tehran, Iran.

2. Department of Occupational Therapy, Rehabilitation Research Center, School of Rehabilitation, Iran University of Medical Sciences, Tehran, Iran.

\begin{tabular}{|c|c|}
\hline $\begin{array}{l}\text { Use your device to scan } \\
\text { and read the article online }\end{array}$ & Citation Soltaninejad, H., Alizadeh Zarei, M., Akbarfahimi, M., Azad, A., \& Rabiei, F. (2021). The Development and Valida- \\
\hline 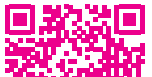 & $\begin{array}{l}\text { tion of a Scale for the Activities of Daily Living in Iranian Children. Basic and Clinical Neuroscience, 12(6), 837-848. http:// } \\
\text { dx.doi.org/10.32598/bcn.2021.2732.1 }\end{array}$ \\
\hline 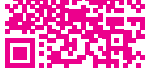 & doil http://dx.doi.org/10.32598/bcn.2021.2732.1 \\
\hline
\end{tabular}

\section{(1) (\$)}

\section{Article info:}

Received: 10 Jul 2020

First Revision: 15 Aug 2020

Accepted: 10 Oct 2020

Available Online: 01 Nov 2021

Keywords:

Scale development, Activities of daily living, Children,

Outcome measure

\section{AB S T RAC T}

Introduction: Activities of Daily Living (ADL), as an ultimate goal of rehabilitation, rely on cultural and environmental factors. This study aimed to develop a questionnaire based on the occupational therapy practice frame to accurately evaluate Iranian children's occupational performance.

Methods: This scale was developed in two phases of planning and construction. The planning phase involved a literature review and a collection of the available evaluation tools in the area. The advice of two expert panels was used to develop a preliminary 87 -item questionnaire. In the construction phase, 40 parents were surveyed to assess the popularity of the activities in Iran. After a face to content validation, the final version of the questionnaire was prepared with 93 items.

Results: The final 93-item questionnaire was used to assess the ADL of 3-6-year-old children. The 93 items, selected according to criteria found in the literature and the panel of experts, were categorized into six ranges of occupational therapy practice framework (bathing/washing/ personal hygiene, toileting, dressing, eating/feeding, functional mobility, and others).

Conclusion: The ADL in Iranian children is a practical and culturally relevant tool for measuring the occupational performance of Iranian children. It can be used in clinical and population-based research.

\footnotetext{
* Corresponding Author:
}

Mehdi Alizadeh Zarei, PhD.

Address: Department of Occupational Therapy, Rehabilitation Research Center, School of Rehabilitation, Iran University of Medical Sciences,

Tehran, Iran.

Tel: $+98(912) 3070065$

E-mail:mehdii.alizadeh@yahoo.com 


\section{Highlights}

- ADLs are essential and routine tasks required to independent and healthy living.

- The active participation of the child in ADL during development has a positive effect on the development of sensory and cognitive functions of the brain.

- ADLs are highly dependent on contextual and ecological factors, such as parenting style, the environment and given context.

- The ADLIC tool is a standard, culturally adapted tool that can be used by occupational therapists to assess ADLS in Iranian children.

\section{Plain Language Summary}

The ADLs referred to Self Care Skills, which play a major role in a child overall functional growth, confidence and independence. The healthy individuals can perform these life skills without assistance. The inability to accomplish essential ADLs may lead to unsafe conditions and poor quality of life. Due to the importance of these activities during the development of the child, it is necessary for Iranian children to be evaluated in terms of independence in these activities so that in case of disorder, the necessary counseling and education can be provided by occupational therapists.

\section{Introduction}

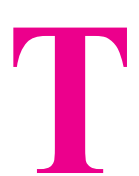

he first and most essential occupational performance area in daily living activities, like self-care, can be marked as the most crucial. This area also includes all the skills a person needs to manage basic activities in their life (Law, Baum, \& Dunn, 2017). The occupational performance addresses the needs in life resulting from the complex interconnections between the individual as an open system and the environment in which activities, tasks, and roles occur (Smith, 2013). Activities of Daily Living (ADL) in the Occupational Therapy Practice Frame (OTPF) include 10 activities: bathing/showering, donning and doffing clothes, eating, feeding, functional mobility, personal care, personal hygiene, and grooming, sexual activity, and toilet care (American Occupational Therapy Association, 2014). Occupational therapists use the activities as therapeutic interventions to educate various skills to their clients. The ultimate goal of occupational therapy is to enhance the ability of clients to achieve independent living, regardless of the diagnostic and therapeutic use of the activities and occupations (Richardson, 2013).

Neuro-occupation is a model that incorporates the system theory and is built from the combination of occupation and neuroscience. It demonstrates that the relationship between the nervous system and the participation of people in occupation is non-linear. Neuro-occupation is the connection between the brain nervous system and aims to achieve a meaningful career (Derakhshanrad et al., 2015; Gutman, \& Biel, 2001; Dehaqani et al., 2016).

Neuro-occupation states that voluntary participation of the person in ADL results in creating meaning for that person. Considering this model, the occupation selected by the individual and the environment in that the person acts can influence brain function (Lazzarini, 2004).

Non-Linear relevance between parts of the organization in the dynamic system influence outcomes and result in self-organization. This fact is related to the system's capacity to manage its activities. Therefore, the system manages its act independently to be attentive to the requirements encountered by the system in the best conceivable manner. This property permits the system to come up with changes. This new phenomenon, called bio-dynamic or non-linear dynamic biology, explains cellular and molecular biology's vague components to neuroscience (Mehdizadeh, Hassani Mehraban, \& Zahediyannasab, 2017).

The quality of the brain self-organization may be affected by the two-way relationships between participation in occupation and the neuro-dynamic system (Derakhshanrad et al., 2015; Lazzarini, 2004). 
ADL that affect performance highly depends on contextual and ecological factors, such as child care, the environment, a given context, specific aspects of self-care, and child functional skills that enhance a child's ability to pair successfully ticipate in ADL (Smith, \& Clifford, 2013; Pashmdarfar \& Azad, 2020).

The ADL are performed in internal and external situations. Internal situations can include body functions and structures related to disability and cultural and individual contexts. In contrast, external situations may include physical and social environments and spiritual and cultural aspects of the environment. An environment affects the demands of activity; these may consist of changes in object use, social and spatial demands, sequences and timing, required actions, body functions, and body structures (Case-Smith, \& Clifford O’Brien, 2013).

Factors affecting adequacy and competence in ADL are related to the child's functional skills, such as motoric capability, sensory system, and cognitive functioning. Lifestyle, socioeconomic status, and familial educational level are crucial factors in the type of children's ADL. As the child grows up, the habits, patterns of activity, and expectations of others also influence the daily activities of a child's life. Culture is another influencing factor in this regard, as it influences a child's independence in ADL through the availability of equipment and materials (Richardson, 2013).

Adapting to eating utensils (spoons, forks), food preparation, and overall conditions are other cultural factors that will help make the child independent in self-help activities.

Chen, Tseng, Hu, and Koh (2010) evaluated American and Taiwanese children. They found that Taiwanese children were less independent in self-care activities than American children, especially after the age of 4, and required further support and care from their parents. This is mainly due to the overwhelming emphasis of American parents on child independence and participation in daily activities. In contrast, Taiwanese parents are more interested in teaching their children cognitive skills in elementary school.

A review of the existing available tests in Iran (Pediatric Evaluation of Disability Inventory (PEDI), Children Participation Questionnaire (CPQ), Vineland Adaptive Behavior Scale (VABS), Wee Functional Independence Measure (Wee FIM), KLEIN BELL Activities of daily living Scale (KLEIN BELL) revealed that while the ADL assessment is conducted in these tests, none of these tests are per the OTPF framework. It should also be considered that how the child performs ADL is highly culturally dependent. And although the tests may have been translated into Persian, certain items from the tests have not been adapted to Persian culture. Furthermore, the available tests in Iran do not specifically assess ADL components in children of preschool age, which is the age period with the most prevalent functional differences in ADL and reflects parental attitudes and parenting patterns arising from cultures.

This research evaluated 3-6-year-old children because this age range provides a slightly different early childhood experience concerning activity requirements, environmental factors, and family considerations from its previous infancy stages and later late and middle childhood stages. Therefore, a tool that can specifically assess clients within the Iranian culture by considering all components of ADL was needed (Case Smith, \& Clifford O'Brien, 2015). Accordingly, this study has developed an OTPF based scale for occupational performance in 3-6-year-old children.

\section{Methods}

The phases of scale development phases were employed to develop this instrument. This instrument was developed in two phases, as elaborated below (Benson, \& Clark, 1982; Amini, Hassani Mehraban, Haghni, Asgharnezhad, \& Khayatzadeh Mahani, 2016).

Phase 1, or the planning phase includes two steps: the target population and purpose are identified in step 1 . In step 2, the literature is reviewed, and an item pool is created using open-ended questions. Phase 2, or the construction phase, includes steps 3 (study survey), 4 (statistical analysis), and 5 (content $\&$ face validity).

Phase I: Designing the scale (Appendix 1)

Step 1: The target population and declaration of the purpose of the study. This study aimed to generate an instrument for assessing the activities of daily living of Iranian children aged 2 to 6 years.

Step 2: Literature review and item pool preparation using open-ended questions. A critical review was then conducted on the existing instruments for children's ADL assessment. Items from the available instruments such as PEDI, CPQ, VINELAND, WeeFIM, and KLEIN BELL were integrated and combined, and an item pool with an activity set of 324 items was devised. Afterward, an expert panel (4 occupational therapists, 2 neurosci- 
entists, 1 pediatric psychologist) was held. According to the child case report, the inclusion criteria for the participants were being in the age range of 3 to 6 years and having had healthy development and motor skills. The panel mentioned above aimed to investigate the frequency of the activities in Iran and to check the face validity of the items by considering the Iranian community.

Following the panel discussion, the specialists first determined 6 main fields of the test (bathing/ washing/personal hygiene, toileting, dressing, eating/feeding, functional mobility, and others), i.e., merged from 9 fields of ADL. The experts merged and combined many items to reduce the number, which came down to an activity set with 107 items.

A second expert panel was held to review the obtained items, check the face validity of the items, and allocate activities to the areas of occupation in OTPF [ADL, Instrumental Activities of Daily Living (IADL), play, leisure, social/participation, education, work, sleep, and rest] (American Occupational Therapy Association, 2014). The experts in both panels were the same. Two items were removed in the first panel and replaced by 4 other items, and some items were merged. The final product delivered a set of activities with 87 items. In phase I, we successfully developed an appropriate questionnaire with 87 items, including an activity set in conjunction with open-ended questions.

\section{Phase II: Construction}

Step3: Survey study. In this step, a survey study was obtained from 40 parents of 2-6-year-old children (average age: 3.94 , SD: 1.28 ) via the questionnaire developed in phase 1 . these children were selected from different kindergartens in Tehran using the convenience sampling method. Approximately $87.5 \%$ of them lived in an apartment, and $12.5 \%$ lived in a house (Table 1).

The selection was based on maximum diversity in lifestyles and family education. The parents were interviewed for this study and were requested to recommend any other familiar activities. The participants were also requested to respond with 'yes' or 'no' to the questions in the questionnaire. Sampling was performed in kindergarten settings. This survey aimed to determine the frequent activities of Iranian children.

Step 4: In this stage, the statistical analysis of the survey study's information was done to identify the cut-off scores. In these studies, the proportion ratio equals $60 \%$ 80\% (Serra Sutton et al., 2009; Alvik, \& Grøholt, 2011), the gained ratio considered for containing the items was $70 \%$ (i.e., $70 \%$ of participants completed the activity) (Serra Sutton et al., 2009; Alvik, \& Grøholt, 2011). A third expert panel was held to assess the pilot study results and discuss the participants' responses. Face validity was also evaluated in this panel. Based on the pilot study results and the discussion within panel 3,2 items were omitted, and 14 items were added by expanding 6 items, bringing the total to a set of activities with 93 items.

\section{Step 5: Content and face validity}

Content and face validity were analyzed in this stage. The Content Validity Index (CVI) and Content Validity Ratio (CVR) were calculated after consulting with 15 occupational therapists to examine content validity. The impact score was calculated to examine face validity. The calculation process is explained below:

\section{Face validity evaluation}

In this study, 60 parents were surveyed for face validity, and consent forms were received from the parents. Imact score was used to measure the importance of these items. A Likert scale with 5 options and scores of 1-5 from critical (score 5), important (score 4), standard important (score 3), slightly important (score 2), and not significant (score 1) was considered and rated for each item. If the impact score of an item was $>1.5$, the item was considered suitable for the subsequent analysis and was preserved (Yaghmaei, 2003; Hosseini, Ghorbani, \& EBN Ahmady, 2015) (Equation1).

Equation1. The route used to calculate the impact score:

\section{Impact Score $=$ Frequency $(\%) \times$ Importance}

\section{Content validity evaluation}

The CVI and the CVR were measured to assess the content validity of the test.

\section{CVI}

This test was given to 15 occupational therapists (with $\mathrm{PhD}$ degrees) with a mean of twenty years of experience in assessment and clinical interventions to evaluate its content validity index. Each item of the questionnaire was assessed according to three concepts: simplicity, relevance or specificity, and clarity. Simplicity was evaluated on a scale of 1 (complicated), 2 (needs modification), 3 (simple but needs reviewing), and 4 (straightforward). The relevance of each item was evaluated on a scale of 1 (not relevant), 2 (somewhat relevant), 3 (entirely appropriate), and 4 (highly 
Table 1. The frequency distribution of the demographic data

\begin{tabular}{ccc}
\hline & Variables & No. (\%) \\
\hline \multirow{2}{*}{ Gender } & Male & $23(57.5)$ \\
& Female & $17(42.5)$ \\
\hline \multirow{2}{*}{ Residence type } & Apartment House & $35(87.5)$ \\
Ownership & Owner Tenant & $5(12.5)$ \\
& & $26(65)$ \\
Mother's education & Secondary school's degree & $14(35)$ \\
& Diploma & $1(2.5)$ \\
& Associate's degree & $4(2.5)$ \\
& Bachelor & $7(10)$ \\
Father's education & Master's PhD. & $21(17.5)$ \\
& Secondary school's degree & $5(52.5)$ \\
& Diploma & $2(12.5)$ \\
& Associate's degree & $3(7.5)$ \\
& Bachelor & $8(20)$ \\
& Master's PhD. & $3(7.5)$ \\
& Clerk & $14(35)$ \\
Father's job & Housewife & $8(20)$ \\
& Clerk & $4(10)$ \\
\hline \multirow{2}{*}{ Mother's job } & Self-employed & $13(32.5)$ \\
& & $27(67.5)$ \\
\hline
\end{tabular}

NEUR SCIENCE

relevant). The item clarity was evaluated according to 1 (not clear), 2 (somewhat clear), 3 (quite clear), and 4 (highly clear). After completing all the aforementioned processes, the quantitative CVI was calculated employing Equation 2. The acceptable amount of CVI was considered 0.79 (Yaghmaei, 2003; Waltz \& Bausell, 1981; Polit, \& Beck, 2006; Polit, Beck, \& Owen, 2007).

Equation 2. The method used to calculate the quantitative CVI:

$C V I=\frac{\text { The Number of the specialists who have checked option } 3 \text { and } 4}{\text { The total number of specialists }}$

CVR: The CVR is based on the Lawshe scale and is used to assess the necessity of each item. Each item was scored according to three options on the graph $(1=$ not necessary, $2=$ useful, but not essential, and $3=$ essential). An item was considered permissible and crucial if more than fifty percent of experts affirmed its essentiality and if the result of that item was more significant than the score of the Lawshe table (0.56) (Yaghmaei, 2003; Lawshe, 1975) (Equation 3).

Equation 3. Method used to calculate the quantitative CVR:

$$
C V R=\frac{n E-N / 2}{N / 2}
$$

\section{Results}

The Mean \pm SD age of the study participants was $3.94 \pm 1.28$ years (age range: $2-6$ years). These participants were from the north, south, west, and east of Tehran City, Iran. About $87.5 \%$ of them lived in an apartment, and $12.5 \%$ lived in a house (Table 1). The data from the survey were analyzed to determine the cut-off score. The acceptable ratio for including the data was $70 \%$ (i.e., $70 \%$ of the participants performed the activity). Items that received a score higher than $45 \%$ in CVR (Yaghmaei, 2003; Lawshe, 1975) were included, and all the 93 items received a score higher than 56\%. Based on CVI, a score $>79 \%$ is considered acceptable (Shi, Mo, \& Sun, 2012), and all the 93 items received a CVI score of over $79 \%$ (the lowest score was $87 \%$, and the highest was 1). According to the impact score, items that received a score higher than 1.5 in impact score were considered sufficient to be included in the questionnaire. Almost all the included items had an impact score of over 1.5 . The only exception was item 51 (tying shoelaces), which had a score of 1.37 and was not omitted from the questionnaire, as per the recommendation of the experts. The highest score in the impact factor was 5. 

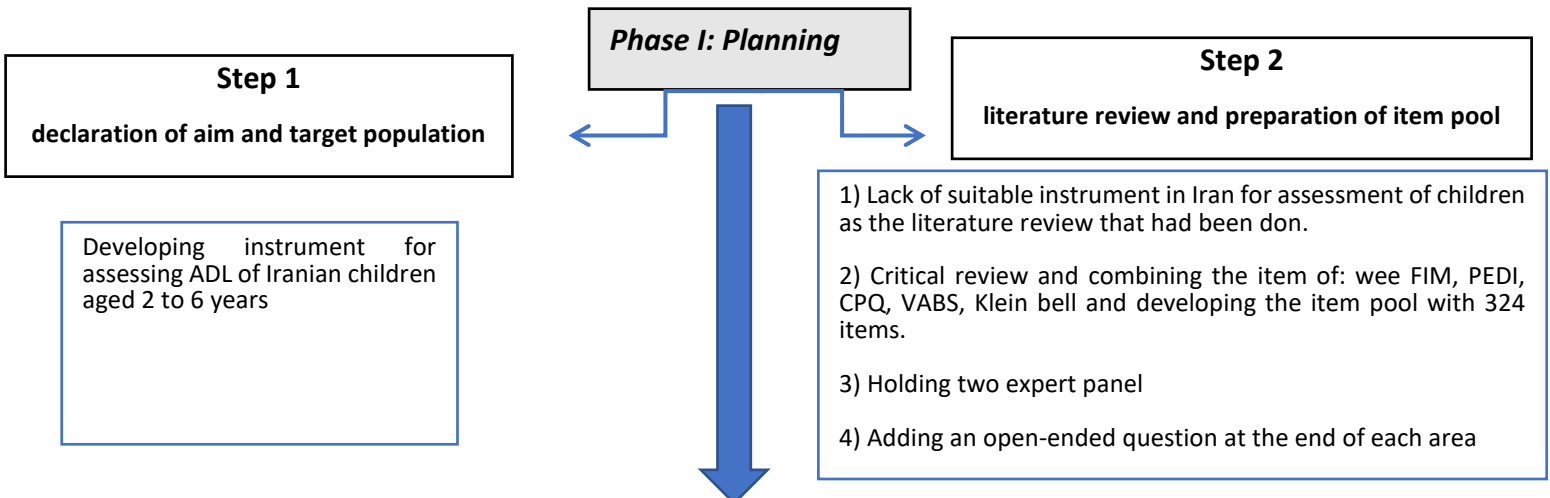

Outcome of Phase I

Developing of a primary questionnaire with 87 items and open-ended question at the end of each area

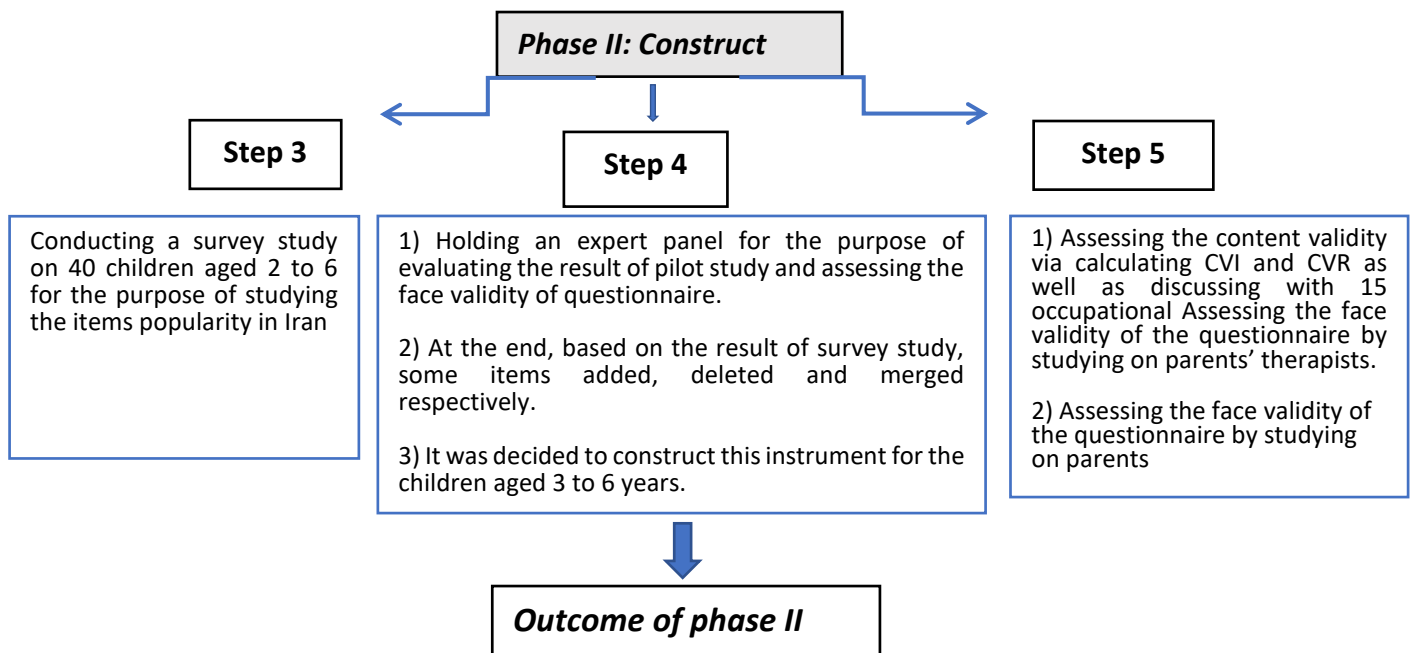

Development of a questionnaire with 93 items

Figure 1.The steps of developing children's ADL assessment scale

NEUR:SCIENCE

\section{Discussion}

This study introduced an instrument for assessing ADL in 3-6-year-old children. While this study had initially been designed to determine ADL in 2-6-year-old children, the obtained results from the pilot study and expert recommendations proved it more suitable for the 3-6-year-old age range. Throughout this study, it was determined that children under the age of 3 (2-3 years old) are highly dependent on parents to perform ADL. Furthermore, there are no accurate tests to evaluate ADL in 2-3-year-old children.

A five-point Likert scale ( $1=$ totally dependent, $2=$ moderately dependent, $3=$ relatively independent, $4=$ adaptively independent, $5=$ totally independent) was used to assess children's dependency in ADL. To develop this instrument and its framework, the OTPF was used, and some areas of ADL in OTPF (bathing/showering, donning \& doffing of clothes, feeding, eating, functional mobility, personal hygiene, and grooming, personal device care, toilet hygiene) were included in our designed questionnaire. Sexual activity was not included in the questionnaire, which distinguishes this instrument from the other existing scales. In this questionnaire, we tried to solve the drawbacks of other existing questionnaires.

The earlier account states that Taiwanese children (with Asian culture) over the age of 4 were less independent in self-care activities and needed more support and care from their parents (Chen et al., 2010). These findings suggest that in addition to child-related factors, which may be less variable in different countries, other factors such 
as socioeconomic status, family lifestyle, and cultural issues significantly affect how a child performs their ADL.

This study introduces an instrument for assessing children's occupational performance in ADL children aged 3 to 6 years. Initial tests used in Iran are not well adapted to the Iranian cultures and are not per the OTPF. For example, while culture has an essential effect on how a child acts in ADL (Chen et al., 2010; Case Smith, \& Clifford O'Brien, 2015), it is not considered in the current tests that assess occupational performance in ADL. Tests administered in Persian do not assess ADL components for preschool-aged children.

Independence in daily life activities is formed at a young age. The most significant functional difference in this field is related to the pre-school age period, which is due to cultural and territorial differences, parental attitudes, and parenting patterns in terms of independence. This instrument was constructed according to a family-centered approach, and it assesses the occupational performance in ADL from the parents' perspectives. As OTPF was used to construct this instrument, and 6 critical areas of daily living activities (bathing/ washing/personal hygiene, toileting, dressing, eating/ feeding, functional mobility, and others) were included in this questionnaire, which is the most significant merit of this questionnaire in comparison with other current instruments. For example, the PEDI test (Nicholas, \& Case Smith, 1996), a test used in various studies, is very lengthy and thus, requires a long period to complete. The ADLIC as a comprehensive measure covers more domains and takes led time to perform. Furthermore, the ADLIC evaluates occupational performance through daily functional activities. There was limited access to parents and their children together. However, this problem was solved by gathering data from other places where children were alongside their parents.

\section{Conclusion}

In conclusion, the validity of the questionnaire developed in the present research was better than the other available instruments; thus, this scale can be used as a descriptive and evaluative questionnaire in the studies on children with and without disabilities in the Iranian culture.

\section{Ethical Considerations}

\section{Compliance with ethical guidelines}

This study was approved by the Ethical Committee of Iran University of Medical Sciences (Code: IR.IUMS. REC.1398.891).

\section{Funding}

This article was extracted from a $\mathrm{PhD}$. dissertation of the first author at the Department of Occupational Therapy, School of Rehabilitation, Iran University of Medical Sciences, Tehran.

\section{Authors' contributions}

All authors equally contributed to preparing this article.

\section{Conflict of interest}

The authors declared no conflict of interest.

\section{Refrences}

Alvik, A., \& Grøholt, B. (2011). Examination of the cut-off scores determined by the ages and stages questionnaire in a populationbased sample of 6 month old norwegian infants. BMC Pediatrics, 11(117), 1-7. [DOI:10.1186/1471-2431-11-117] [PMID] [PMCID]

American Occupational Therapy Association. (2014). Occupational Therapy practice framework: Domain and process. Maryland: AOTA Press/American Occupational Therapy Association. https:// books.google.com/books?id=9HyToAEACAAJ\&dq=American +Occupational+Therapy+Association

Amini, M., Hassani Mehraban, A., Haghni, H., Asgharnezhad, A. A., \& Khayatzadeh Mahani, M. (2016). Development and validation of Iranian children's participation assessment scale . Medical Journal of Islamic Republic of Iran, 30 (1), 199-209. http:/ / mjiri. iums.ac.ir/article-1-3574-en.html

Benson, J., \& Clark, F. (1982). A guide for instrument developmen and validation. The American Journal of Occupational Therapy, 36(12), 789-800. [DOI:10.5014/ajot.36.12.789] [PMID]

Case Smith, J., \& Clifford O'Brien, J. (2015). Occupational therapy for children and adolescents. Amsterdam: Elsevier. https://books. google.com/books?id=6B6_oAEACAAJ\&dq=Case

Case-Smith, J., \& Clifford O'Brien, J. (2013). Occupational therapy for children. Amsterdam: Elsevier Health Sciences. https://books. google.com/books?id=meVOAQAAQBAJ\&dq=Occu

Chen, K. L., Tseng, M. H., Hu, F. C., \& Koh, C. L. (2010). Pediatric evaluation of disability inventory: A cross-cultural comparison of daily function between Taiwanese and American children. Research in Developmental Disabilities, 31(6), 1590-600. [DOI:10.1016/j.ridd.2010.05.002] [PMID] 
Dehaqani, M. R. A., Zarei, M. A., Vahabie, A. H., \& Esteky, H. (2016). Impairement of perceptual closure in autism for vertex-but not edge-defined object image. Journal of Vision, 16(10), 10. [DOI:10.1167/16.10.10]

Derakhshanrad, S. A. R., Piven, E., Hosseini, S. A., Mohammadi Shahboulaghi, F., Nazeran, H., \& Rassafiani, M. (2015). Exploring the nature of the intention, meaning and perception process of the neuro occupation model to understand adaptation to change. Occupational Therapy International, 23(1), 29-38. [DOI:10.1002/oti.1402] [PMID]

Gutman, S. A., \& Biel, L. (2001). Promoting the neurologic substrates of well-being through occupation. Occupational Therapy in Mental Health, 17(1), 1-22. [DOI:10.1300/J004v17n01_01]

Hosseini, Z., Ghorbani, Z., \& EBN Ahmady, A. (2015). Face and content validity and reliability assessment of change cycle questionnaire in smokers. Journal of Mashhad Dental School, 39(2), 147-54. [DOI:10.22038/jmds.2015.3637]

Law, M. C., Baum, C. M., \& Dunn, W. (2017). Measuring occupational performance: Supporting best practice in occupational therapy. New Jersey: Slack. https://books.google.com/books?id $=$ wOW6jwEACAAJ\&dq=Measuring+occupational+perform

Lawshe, C. H. (1975). A quantitative approach to content validity. Personnel Psychology, 28(4), 563-75. [DOI:10.1111/j.1744-6570.1975.tb01393.x]

Lazzarini, I. (2004). Neuro-occupation: The nonlinear dynamics of intention, meaning and perception. British Journal of Occupational Therapy, 67(8), 342-52. [DOI:10.1177/030802260406700803]

Mehdizadeh, M., Hassani Mehraban, A., \& Zahediyannasab, R. (2017). The Effect of group-based occupational therapy on performance and satisfaction of stroke survivors: Pilot trail, neuro occupational view. Basic and Clinical Neuroscience, 8(1), 69-76. [DOI:10.15412/J.BCN.03080109] [PMID] [PMCID]

Nicholas, D. S., \& Case Smith, J. (1996). Reliability and validity of the pediatric evaluation of disability inventory. Pediatric Physical Therapy, 8(1), 15-24. [DOI:10.1097/00001577-199600810-00004]

Pashmdarfard, M., Azad, A. (2020). Assessment tools to evaluate Activities of Daily Living (ADL) and Instrumental Activities of Daily Living (IADL) in older adults: A systematic review. Medical Journal of the Islamic Republic of Iran, 34(1), 224-39. [DOI:10.34171/mjiri.34.33] [PMID] [PMCID]

Pendleton, H. M., \& Schultz-krohn, W. (2013). Pedretti's Occupational Therapy - E-Book: Practice skills for physical dysfunction. Amsterdam: Elsevier Health Sciences. https://www. google.com/books/edition/Pedretti_s_Occupational_Therapy_E_Book/BVFPAQAAQBAJ?hl=en

Polit, D. F., \& Beck, C. T. (2006). The content validity index: Are you sure you know what's being reported? Critique and Recommendations, 29(5), 489-97. [DOI:10.1002/nur.20147] [PMID]

Polit, D. F., Beck, C. T., \& Owen, S. V. (2007). Is the CVI an acceptable indicator of content validity? Appraisal and recommendations. Research in Nursing \& Health, 30(4), 459-67. [DOI:10.1002/nur.20199] [PMID]

Richardson, P. (2013). Teaching activities in occupational therapy. In H. M. Pendleton, \& W. Schultz-krohn (Eds.), Pedretti's occupational therapy: Practice skills for physical dysfunction. Amsterdam: Elsevier Health Sciences. https://books.google. $\mathrm{com} /$ books?id=BVFPAQAAQBAJ\&dq=pedretti $\% 27 \mathrm{~s}+$ occu
Serra Sutton, V., Ferrer, M., Rajmil, L., Tebé, C., Simeoni, M C., \& Ravens Sieberer, U. (2009). Population norms and cut-off-points for suboptimal health related quality of life in two generic measures for adolescents: The Spanish VSPA and KINDL-R. Health Qual Life Outcomes, 7(35), 1-9. [DOI:10.1186/1477-7525-7-35] [PMID] [PMCID]

Shi, J., Mo, X., \& Sun, Z. (2012). Content validity index in scale development. Zhong nan da xue xue bao. Yi xue ban. Journal of Central South University (Medical Sciences), 37(2), 152-5. [DOI:10.3969/j.issn.1672-7347.2012.02.007] [PMID]

Waltz, C. F., \& Bausell, R. B. (1981). Nursing research: Design, statistics, and computer analysis. Michigan: University of Michigan. https:/ / books.google.com/books?id=cyJtAAAAMAAJ

Yaghmaei, F. (2003). Content validity and its estimation . Journal of Medical Education, 3(1), 25-7. https://www.sid.ir/en/journal/ViewPaper.aspx?id=33688 
Appendix

Appendix 1. Children activities of daily living assessment scale

\begin{tabular}{|c|c|c|c|c|c|c|}
\hline No. & Basic Activities of Daily Living (ADL) & CVR & R-CVI & C-CVI & S-CVI & IS \\
\hline 1 & Maintain appropriate body position while showering & 0.73 & 0.87 & 0.87 & 0.93 & 4.50 \\
\hline 2 & Open and close the faucet & 1 & 1 & 1 & 1 & 4.85 \\
\hline 3 & Adjust the water temperature & 0.87 & 0.93 & 0.93 & 0.93 & 1.81 \\
\hline 4 & Select and use the bathing tools (soap, shampoos, washcloth) & 0.87 & 1 & 0.93 & 1 & 4.09 \\
\hline 5 & Recognize your toothbrush & 1 & 1 & 1 & 1 & 4.90 \\
\hline 6 & Recognize your comb & 0.73 & 1 & 1 & 1 & 4.85 \\
\hline 7 & Wash hair by shampoo & 1 & 0.93 & 0.93 & 1 & 3.40 \\
\hline 8 & Wash body by washers & 0.87 & 1 & 1 & 1 & 3.44 \\
\hline 9 & Organize washing tools after showering & 0.6 & 0.93 & 0.93 & 1 & 2.59 \\
\hline 10 & Rinse the hair & 0.6 & 1 & 1 & 1 & 4.27 \\
\hline 11 & Rinse the body & 0.87 & 1 & 1 & 1 & 4.37 \\
\hline 12 & Dry the hair & 0.87 & 1 & 1 & 1 & 4.50 \\
\hline 13 & Dry the body & 0.87 & 1 & 0.93 & 1 & 4.22 \\
\hline 14 & Open and close the lid of toothpaste & 0.87 & 1 & 1 & 1 & 4.41 \\
\hline 15 & Hold the toothbrush and put the toothpaste on it & 0.87 & 1 & 1 & 1 & 3.82 \\
\hline 16 & Brush the teeth & 0.87 & 0.93 & 0.93 & 0.93 & 4 \\
\hline 17 & Wash hands & 0.87 & 0.87 & 0.8 & 0.8 & 3.86 \\
\hline 18 & Wash the brush and put it in the correct location & 0.73 & 1 & 1 & 1 & 3.56 \\
\hline 19 & Use the washers to rinse face and hands & 1 & 0.93 & 0.93 & 1 & 4.05 \\
\hline 20 & Dry the face and hands & 0.87 & 1 & 1 & 1 & 4.60 \\
\hline 21 & Use the towel/cloth to clean or wipe out & 0.6 & 1 & 1 & 1 & 3.91 \\
\hline 22 & Comb and tidy the hair & 0.73 & 1 & 1 & 1 & 2.92 \\
\hline 23 & Hold a tissue in front of the nose while coughing and sneezing & 0.87 & 1 & 1 & 1 & 3.61 \\
\hline 24 & Clean the nose and mouth by the tissue & 1 & 1 & 1 & 0.87 & 4.23 \\
\hline 25 & Blow the nose & 0.73 & 1 & 1 & 1 & 3.32 \\
\hline 26 & Maintain appropriate hand and foot position for cutting the nails & 0.73 & 0.93 & 0.93 & 1 & 3.44 \\
\hline 27 & $\begin{array}{l}\text { Demonstrate the need to change wet or dirty diapers when urinating } \\
\text { and defecating }\end{array}$ & 0.73 & 1 & 1 & 1 & 4.90 \\
\hline 28 & Demonstrate the need to go to the toilet for defecating & 0.73 & 1 & 1 & 1 & 4.51 \\
\hline 29 & Find the location of the restroom & 1 & 1 & 1 & 1 & 4.95 \\
\hline 30 & Go and come back from the toilet & 0.87 & 1 & 1 & 0.93 & 4.41 \\
\hline
\end{tabular}




\begin{tabular}{|c|c|c|c|c|c|c|}
\hline No. & Basic Activities of Daily Living (ADL) & CVR & R-CVI & C-CVI & S-CVI & IS \\
\hline 31 & Pull down the pants before toileting & 0.87 & 1 & 1 & 1 & 4.09 \\
\hline 32 & Take off the pants/underwear before toileting & 0.73 & 1 & 1 & 1 & 4.65 \\
\hline 33 & Get the right position for toileting & 0.87 & 1 & 1 & 1 & 4.05 \\
\hline 34 & Get the right position for using Iranian toilet & 1 & 1 & 1 & 0.87 & 4.27 \\
\hline 35 & Finish the action of defecation & 0.87 & 1 & 1 & 1 & 4.41 \\
\hline 36 & Wash and clean yourself after urination & 0.73 & 1 & 1 & 1 & 3.48 \\
\hline 37 & Wash and clean yourself after defecation & 0.87 & 1 & 1 & 1 & 3.28 \\
\hline 38 & Wash the toilet after defecation & 0.73 & 1 & 1 & 1 & 2.85 \\
\hline 39 & Wash hands with water and detergents after defecation 1 & 0.87 & 1 & 1 & 1 & 4.09 \\
\hline 40 & Wear your pants after toileting & 1 & 1 & 1 & 1 & 4.46 \\
\hline 41 & Pull up your pants after toileting & 1 & 1 & 1 & 1 & 4.65 \\
\hline 42 & Dry clothes or diapers continuously during the day & 0.73 & 1 & 0.87 & 0.93 & 4.18 \\
\hline 43 & Dry clothes or diapers continuously during at night & 0.6 & 1 & 0.87 & 0.93 & 4.46 \\
\hline 44 & Identify "on" and "under" of the clothes & 0.87 & 1 & 1 & 1 & 3.78 \\
\hline 45 & Identify "back" and "front" of the clothes & 1 & 1 & 1 & 1 & 3.99 \\
\hline 46 & Open the buttons & 0.87 & 1 & 1 & 1 & 3.69 \\
\hline 47 & Close the buttons & 0.87 & 1 & 1 & 1 & 2.83 \\
\hline 48 & Open the zipper, buckle, Velcro & 1 & 1 & 1 & 1 & 4.09 \\
\hline 49 & Close the zipper, buckle, Velcro & 0.87 & 1 & 1 & 1 & 3.07 \\
\hline 50 & Tide the shoelaces & 0.6 & 1 & 0.93 & 1 & 2.13 \\
\hline 51 & Untied the shoelaces & 0.73 & 1 & 1 & 1 & 1.37 \\
\hline 52 & Put off your shoes & 0.73 & 1 & 1 & 1 & 4.41 \\
\hline 53 & Wear your shoes & 1 & 1 & 1 & 1 & 3.91 \\
\hline 54 & Take off the button-through clothes & 1 & 1 & 1 & 1 & 4.09 \\
\hline 55 & Take off the blouse & 1 & 1 & 1 & 1 & 4.14 \\
\hline 56 & Take off the trousers/shorts/knickers/underpants & 1 & 1 & 1 & 1 & 4.56 \\
\hline 57 & Put on the button-through clothes & 1 & 1 & 1 & 1 & 4.09 \\
\hline 58 & Put on the blouse & 0.87 & 1 & 1 & 1 & 3.61 \\
\hline 59 & Put on the shorts/knickers/underpants & 0.87 & 1 & 1 & 1 & 4.09 \\
\hline 60 & Put on the trousers & 1 & 1 & 1 & 1 & 3.96 \\
\hline 61 & Take off the socks & 0.87 & 1 & 1 & 1 & 4.85 \\
\hline 62 & Wear the socks & 0.73 & 1 & 1 & 1 & 3.78 \\
\hline
\end{tabular}




\begin{tabular}{|c|c|c|c|c|c|c|}
\hline No. & Basic Activities of Daily Living (ADL) & CVR & R-CVI & C-CVI & S-CVI & IS \\
\hline 63 & Take off the hat & 0.6 & 1 & 1 & 1 & 5 \\
\hline 64 & Wear the hat & 0.87 & 1 & 1 & 1 & 5 \\
\hline 65 & Swallow the liquids & 0.87 & 1 & 1 & 1 & 5 \\
\hline 66 & Swallow the food without getting stuck in the throat & 1 & 1 & 1 & 1 & 5 \\
\hline 67 & Chew the food & 0.73 & 1 & 1 & 1 & 5 \\
\hline 68 & Put the food in the mouth using the fingers & 0.87 & 1 & 1 & 1 & 4.95 \\
\hline 69 & Use a spoon & 1 & 0.93 & 0.93 & 1 & 5 \\
\hline 70 & Use a fork & 0.6 & 0.93 & 0.93 & 1 & 4.09 \\
\hline 71 & Use a knife & 0.73 & 1 & 1 & 1 & 3.28 \\
\hline 72 & Eat the pureed/crushed food & 1 & 1 & 0.93 & 0.93 & 5 \\
\hline 73 & Eat the solid and mixed (soup, rise, chicken) food & 0.87 & 1 & 0.93 & 0.93 & 4.60 \\
\hline 74 & Drink from the cup or glass & 0.87 & 1 & 1 & 1 & 4.95 \\
\hline 75 & $\begin{array}{c}\text { Roll/ move with buttock on the floor/creep/crawl to browse in the } \\
\text { house }\end{array}$ & 1 & 1 & 1 & 1 & 4.51 \\
\hline 76 & Go to the bed & 0.73 & 1 & 1 & 1 & 5 \\
\hline 77 & Get out of the bed & 0.87 & 1 & 1 & 1 & 5 \\
\hline 78 & Change the position from sleeping to sitting and vice versa & 0.73 & 1 & 1 & 1 & 4.95 \\
\hline 79 & Sand up and sit on the bench & 1 & 1 & 1 & 1 & 4.95 \\
\hline 80 & Stand with maintaining the balance & 0.87 & 1 & 1 & 1 & 4.95 \\
\hline 81 & Walk outside the house & 1 & 1 & 0.93 & 0.93 & 4.60 \\
\hline 82 & Walk on the ramp & 0.73 & 0.93 & 0.87 & 0.93 & 4.80 \\
\hline 83 & Walk on the uneven surface & 0.73 & 1 & 1 & 1 & 4.51 \\
\hline 84 & Go up and down the stairs & 0.87 & 1 & 1 & 1 & 4.90 \\
\hline 85 & Change the way while walking and maintain the balance & 0.87 & 1 & 1 & 1 & 4.95 \\
\hline 86 & Carry the objects while walking & 1 & 1 & 1 & 1 & 4.65 \\
\hline 87 & Move through the spaces inside the house (rooms, kitchen, living room) & 0.73 & 1 & 1 & 1 & 5 \\
\hline 88 & Get in the car & 0.87 & 1 & 1 & 1 & 4.90 \\
\hline 89 & Get out of the car & 0.73 & 1 & 1 & 1 & 4.90 \\
\hline 90 & Replace on the car seat & 0.87 & 1 & 1 & 0.93 & 4.95 \\
\hline 91 & Put their own belongings in place & 0.87 & 1 & 1 & 1 & 4.32 \\
\hline 92 & Demonstrate the interest to change the wet/dirty clothes & 0.73 & 1 & 1 & 1 & 4.90 \\
\hline 93 & Use a pin/head pull to tie the hair & 0.6 & 1 & 1 & 1 & 1.92 \\
\hline
\end{tabular}

S-CVI: Simplicity-Content Validity Index; R-CVI: Relevancy-Content Validity Index; C-CVI: Clarity-Content Validity Index; CVR: Content Validity Ratio. 
This Page Intentionally Left Blank 